\title{
Association of rs10811656 on 9P21.3 with the risk of coronary artery disease in a Chinese population
}

\author{
Jianjun Yan ${ }^{1,2+}{ }^{+}$Jinmei Zeng ${ }^{3+}$, Zhiyong Xie ${ }^{1}$, Dongchen Liu', Liansheng Wang ${ }^{1 *}$ and Zhong Chen ${ }^{3 *}$
}

\begin{abstract}
Background: Genome-wide association studies have reported that the 9p21.3 locus confers risk for coronary artery disease (CAD). However, it is not known whether rs10811656 is linked with CAD in a Chinese population. Thus, the purpose of this study was to investigate the potential association between rs10811656 and the risk of CAD in a Chinese population.

Methods: We conducted a hospital-based, case-control study with 251 CAD patients and 304 controls to examine the potential association of rs10811656 with CAD.

Results: The frequencies of the $\Pi$ genotypes in CAD cases were significantly different from those in controls (adjusted OR: 1.96, $95 \%$ Cl: 1.09-3.505, $P=0.024$ ). Compared to controls, rs 10811656 was significantly associated with the stable angina pectoris (adjusted OR: 1.42, $95 \% \mathrm{Cl}: 1.06-1.90, P=0.017$ ), but not with acute coronary syndrome. There was also a highly significant association of rs 10811656 with double-vessel and triple-vessel disease when patients were divided into subgroups based on the number of diseased vessels (adjusted OR: 1.68 and 1.60, $95 \%$ Cl: $1.14-2.44$ and $1.10-2.33, P=0.009$ and 0.02 , respectively).
\end{abstract}

Conclusion: Our results suggest that the rs10811656 locus might be associated with CAD in a Chinese Han population.

Keywords: SNP, single nucleotide polymorphism, Association, rs10811656, CAD, coronary artery disease, Susceptibility

\section{Background}

Coronary artery disease (CAD) is one of the major causes of morbidity and mortality throughout the world, including China [1, 2]. Epidemiological studies have identified several risk factors for CAD, including dyslipidaemia, diabetes, obesity, smoking and genetic variance [1]. Most recently, genomics analyses have revealed a series of new candidate markers that may contribute to the pathogenesis of CAD [3].

\footnotetext{
*Correspondence: drlswang@njmu.edu.cn; zhongchen7498@sina.com.cn ${ }^{\dagger}$ Equal contributors

'Department of Cardiology, the First Affiliated Hospital of Nanjing Medical University, Nanjing 210029, China

${ }^{3}$ Department of Cardiology, Shanghai Jiao Tong University Affiliated Sixth People's Hospital, East Campus, No. 222, Huanhu Xisan Road, Pudong New Area, Shanghai 201306, China

Full list of author information is available at the end of the article
}

The $9 \mathrm{p} 21.3$ risk locus spans a $>50-\mathrm{kb}$ genomic region and comprises 59 linked single nucleotide polymorphisms (SNPs) that are in strong linkage disequilibrium. This genomic region encodes the cyclin-dependent kinase inhibitors, CDKN2A (p16 and p14) and CDKN2B (p15) [4]. Recently, genome-wide association studies have shown that common variants in the $9 \mathrm{p} 21.3$ gene desert might be robustly associated with the risk of $\mathrm{CAD}$, acute coronary syndrome (ACS), early atherosclerosis, abdominal aortic aneurysm, and intracranial aneurysm [5-7], although the mechanisms underlying these associations are not completely understood. However, it remains unknown whether rs10811656 polymorphism in this region might be linked with the risk of CAD.

The objective of the present hospital-based, case-control study was to investigate the potential association 
between rs10811656 and the risk of CAD, and further replicate the positive association of rs10811656 and ACS in a Chinese population.

\section{Methods}

\section{Study population}

This association study consecutively enrolled 251 unrelated consecutive CAD patients, who were all admitted to Shanghai Jiao Tong University Affiliated Sixth People's Hospital, East Campus from January 2014 to December 2015. CAD was defined as $\geq 50 \%$ luminal narrowing in at least one coronary artery. Patients with CAD were divided into subgroups with 1-vessel, 2-vessel or 3-vessel disease according to the number of significantly affected vessels using the Coronary Artery Surgery Study classification [8]. Two cardiologists who were blinded to all patient information assessed the angiograms. Control subjects $(n=304)$, who underwent coronary angiography to rule out $\mathrm{CAD}$, were also enrolled during the same time period at the same hospital. All subjects enrolled in this study were of Han Chinese origin and had no history of significant concomitant diseases, including cardiomyopathy, bleeding disorders, renal failure, thyroid disease, pulmonary hypertension, and malignancy. This study was approved by the Ethics Committee of Shanghai Jiao Tong University Affiliated Sixth People's Hospital, East Campus and informed consent was obtained from each participant. All authors had no access to information that could identify individual participants after data collection.

\section{DNA extraction and genotyping}

Peripheral venous blood was drawn from each participant in the first day after admission. Genomic DNA was extracted using the AxyPrep DNA Blood kit (Axygen Scientific Inc, Union City, CA, USA). The rs10811656 polymorphism was genotyped by PCR-LDR sequencing method, as reported previously [9].

\section{Statistical analysis}

The allele frequencies and genotype distributions of the CAD cases and controls were compared using chisquare $(x 2)$ tests. Among the controls, the genotype frequencies were tested using the $\chi 2$-test test for the Hardy-Weinberg equilibrium (HWE). The association between the genotypes and CAD was evaluated by computing odds ratios (ORs) and $95 \%$ confidence intervals (95\% CIs). A multiple logistic regression model was used to adjust for covariates including age, sex, body mass index, smoking status, hypertension, diabetes, and dyslipidemia. The linear trend in the association of rs10811656 polymorphism with CAD severity was evaluated by the $\chi 2$-test for trend. Two-tailed $\mathrm{P}$ values $<0.05$ were considered significant. All of the statistical analyses were performed using SPSS for Windows version 13.0 (SPSS, Inc., Chicago, USA).

\section{Results}

Demographic information

The baseline characteristics of the case and control subjects are presented in Table 1. Patients with CAD were older and had higher rate of smoked and diabetes compared to the control subjects. Based on coronary angiography, 133 (43.75\%) patients in the CAD group had single-vessel disease, 85 (27.97 \%) had double-vessel disease and 86 (28.28 \%) had triple-vessel disease.

\section{Genotypes and allele frequencies in CAD cases and controls and their associations with CAD}

The minor allele $(\mathrm{T})$ frequency of rs10811656 was 0.3817 , and the genotype distribution in our study subjects showed no deviation from the Hardy-Weinberg equilibrium $(P>0.05)$. The distribution of the $\mathrm{T}$ genotype was also markedly different between CAD patients and controls $(P=0.024)$, as shown in Table 2. After adjustment for age, sex, hypertension, diabetes, and dyslipidemia, the OR for CAD in subjects with the variant genotypes (CT and TT) was 1.43 (95 \% CI: 0.98-2.08, $P$ $=0.057$ ). We also found that homozygous TT carriers had a $96 \%$ increase in risk of CAD compared with CC carriers (95\% CI: $1.09-3.505, P=0.024)$. In the subgroup of patients with stable angina pectoris (SAP), carriers of TT homozygous carriers had a $42 \%$ increase in the risk of CAD compared with the CC carriers (95\% CI: 1.06-1.90, $P=0.017)$. However, no significant association was observed with ACS (Table 3.).

Table 1 Baseline characteristics of the study subjects

\begin{tabular}{llll}
\hline Characteristic & Controls $(n=251)$ & CAD cases $(n=304)$ & $P$ values \\
\hline Age (years) & $63.0 \pm 14.5$ & $67.13 \pm 11.7$ & $<0.001$ \\
Sex (male), n (\%) & $47(39.8)$ & $127(63.8)$ & $<0.001$ \\
Hypertension, n (\%) & $73(61.9)$ & $141(71.2)$ & 0.086 \\
Diabetes, n (\%) & $16(13.6)$ & $65(32.7)$ & $<0.001$ \\
Dyslipidemia, n (\%) & $39(33.1)$ & $76(38.2)$ & 0.358 \\
Smoking, n (\%) & $23(19.5)$ & $71(35.7)$ & 0.002 \\
TC (mmol/L) & $4.57 \pm 1.70$ & $4.41 \pm 1.26$ & 0.21 \\
TG (mmol/L) & $1.94 \pm 5.35$ & $1.69 \pm 2.02$ & 0.45 \\
HDL-C (mmol/L) & $1.18 \pm 0.30$ & $1.15 \pm 0.27$ & 0.23 \\
LDL-C (mmol/L) & $2.81 \pm 076$ & $2.69 \pm 0.86$ & 0.09 \\
Number of diseased vessels & - & $85(27.97 \%)$ & - \\
One, n (\%) & - & $86(28.28 \%)$ & - \\
Two, n (\%) & - & $133(43.75 \%)$ & - \\
Three n (\%) & & & -
\end{tabular}

$H D L-C$ high density lipoprotein cholesterol, $L D L-C$ low density lipoprotein cholesterol, TC total cholesterol, TG triglyceride 
Table 2 Distribution of genotypes and the CAD risk estimates for rs 10811656

\begin{tabular}{|c|c|c|c|c|c|c|c|}
\hline & & Controls $(n=243)$ & Cases $(n=298)$ & Crude OR (95 \% CI) & $P$ & Adjusted $^{\mathrm{a}}$ OR $(95 \% \mathrm{Cl})$ & $P$ \\
\hline & Genotyp & (\%) & & & & & \\
\hline \multirow[t]{4}{*}{ rs10811656 } & CC & $99(40.7)$ & 103 (34.6) & 1.00 & & 1.00 & \\
\hline & CT & 118 (48.6) & $147(49.3)$ & $1.19(0.83-1.72)$ & 0.33 & 1.32 (0.89-1.94) & 0.164 \\
\hline & $\pi$ & $26(10.7)$ & $48(16.1)$ & $1.77(1.02-3.08))$ & 0.041 & $1.96(1.09-3.505)$ & 0.024 \\
\hline & $C T+T T$ & $144(59.3)$ & $195(65.4)$ & $1.30(0.92-1.84)$ & 0.14 & $1.43(0.98-2.08)$ & 0.057 \\
\hline
\end{tabular}

Cl confidence interval, $O R$ odds ratio

${ }^{a}$ Adjusted for age, sex, body mass index, smoking status, hypertension, diabetes, dyslipidemia

\section{Stratified analyses of the polymorphism and CAD}

Stratified analyses were conducted according to age, gender, and smoking status (Table 4). We noted that $\mathrm{T}$ allele was significantly associated with CAD in males (adjusted OR: 1.82, $95 \% \mathrm{CI}: 1.10-3.01, P=0.02$ ) and smokers (adjusted OR: 2.13, 95 \% CI: 1.06-4.26, $P=$ 0.033), but not in females (adjusted OR: 1.09, $95 \% \mathrm{CI}$ : 0.62-1.91, $P=\mathrm{NS}$ ) or non-smokers (adjusted OR: 1.21, $95 \%$ CI: $0.77-1.89, P=$ NS). Patients with CAD were subclassified into 3 subgroups (single-, double- and triple-vessel disease) based on the number of stenotic coronary arteries. The frequency of patients with $\mathrm{T}$ variant genotypes increased from double- to triple-vessel disease, but not with single-vessel disease (Table 5).

\section{Lipid profiles of CAD cases and controls in the rs10811656 genotypes}

Table 6 shows lipid profiles according to the rs10811656 genotypes in the CAD and control groups. We did not find a significant association between the rs10811656 genotypes and serum triglycerides, HDL-C or LDL-C levels in these two groups.

\section{Discussion}

The present case-control study demonstrated that the homozygous TT genotype of rs10811656 on chromosome 9p21.3 increased the risk of CAD in a Chinese population. The $9 \mathrm{p} 21.3$ risk locus of the genes encoding the cyclin-dependent kinase inhibitors, CDKN2A (p16 and p14) and CDKN2B (p15), are known as suppressors that control cell cycle and cellular senescence $[10,11]$. Moreover, smooth muscle cells homozygous for the 9p21.3 risk alleles showed reduced expression of p16 and $\mathrm{p} 15$, thus increasing proliferation and causing failure to enter senescence in several studies $[4,10]$. In addition, homozygous carriers of the 9p21 risk allele exhibit upregulation of gene sets involved in cellular proliferation of white blood cells [12].

Harismendy et al. demonstrated that the 9p21.3 locus alters CAD risk by modulating inflammatory signaling through altered STAT1 binding and may modify immune responses by regulating expression of IFN, a 21 or related type I IFN; and this in turn affects the expression of the cyclin-dependent kinase inhibitor gene, CDKN2A/B, which is important regulator of cell proliferation, aging, and apoptosis [13]. Furthermore, in the primary HAoSMCs homozygous TT genotype of rs10811656, reduced expression of $\mathrm{p} 16$ and p15disrups TEAD factor binding and TEAD3-dependent transforming growth factor $\beta$ induction of $\mathrm{p} 16$, indicating the TT genotype of rs10811656 could be a functional variant [14].

In the present study, we observed that the distribution of $\mathrm{T}$ genotype was also markedly different between CAD patients and controls After adjusting for risk factors including age, sex, smoking status, hypertension, diabetes, and dyslipidemia, the OR for CAD in subjects with the variant genotypes (CT and TT) was 1.43 , which was of borderline significance $(P=0.057)$. We also found that homozygous TT carriers had a $96 \%$ increase in the risk of CAD compared with the CC carriers $(P=0.024)$. In addition, TT genotype carriers had a $42 \%$ increased risk of SAP (95 \% CI: 1.06-1.90, $P=0.017$ ); however, the risk of ACS was not significantly increased. Our results are not consistent with the observations of Qiang et al. [15]. They reported that there was a higher risk of ACS in subjects with than without the rs10811656 T allele [15].

Table 3 Association of rs10811656 with SAP and ACS

\begin{tabular}{|c|c|c|c|c|c|c|}
\hline SNP & Groups & Genotype & Crude OR (95\% Cl) & $P$ & Adjusted OR $(95 \% \mathrm{Cl})^{\mathrm{a}}$ & $P$ \\
\hline \multirow[t]{4}{*}{ rs10811656 } & & $\mathrm{CC} / \mathrm{CT} / \mathrm{TT}$ & & & & \\
\hline & Control & $99 / 118 / 26$ & 1 & & 1 & \\
\hline & SAP & $66 / 99 / 36$ & $1.36(1.02-1.79)$ & 0.03 & $1.42(1.06-1.90)$ & 0.017 \\
\hline & ACS & $34 / 48 / 12$ & $1.16(0.81-1.65)$ & 0.41 & $1.27(0.87-1.85)$ & 0.21 \\
\hline
\end{tabular}

ACS acute coronary syndrome, $\mathrm{Cl}$ confidence interval, $O R$ odds ratio, SAP stable angina pectoris

${ }^{a}$ Adjusted for age, sex, body mass index, smoking status, hypertension, diabetes, and dyslipidemia 
Table 4 Stratification analysis by age, sex and smoking statue for the association of rs10811656 with CAD

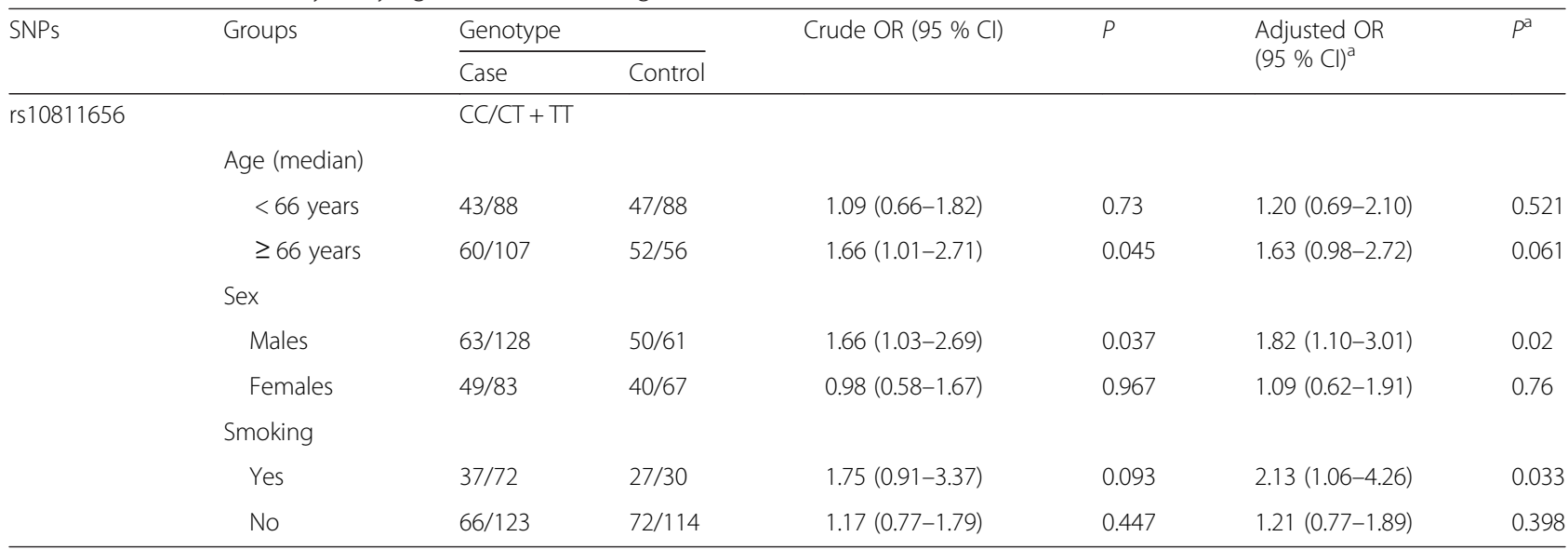

MAF rs10811656 T $=38.17 \%, \mathrm{P}_{\text {for } \mathrm{HW}}=0.379793$

$\mathrm{Cl}$ confidence interval, $\mathrm{OR}$ odds ratio

${ }^{a}$ Adjusted for age, sex, body mass index, smoking status, hypertension, diabetes and dyslipidemia

Studies examining the association of disease severity with the 9p21 genotype are conflicting $[15,16]$. Anderson et al. and Chen et al. found that the 9p21.3 allele predicted the prevelance of CAD, but it did not predict its severity $[16,17]$. The investigation by Muendlein et al. showed that the variant rs1333049 was associated with angiographically characterized coronary atherosclerosis instead of plaque instability, rupture, and thrombogenesis [18]. However, in a study of 769 patients, Ye et al. demonstrated that $9 \mathrm{p} 21$ is associated with a higher prevalence and 5-year progression of preclinical atherosclerosis in the carotid vessels, suggesting that 9p21 promotes plaque formation [19]. Although we did not observe a significant relationship between the rs10811656 T allele and ACS, we found that the frequency of patients with T-variant genotypes increased from double- to triple-vessel disease, whereas there was no association with single-vessel disease. Buysschaert et al. demonstrated that certain alleles on the 9p21 locus are associated with a predisposition to recurrent myocardial infarction and cardiac death within 6 months after the primary event [20].

Associations of SNPs on chromosome 9p21.3 with cardiovascular disease may reflect an influence on the development and progression of coronary atherosclerosis in native arteries rather than causing acute rupture of vulnerable plaques and thrombosis. Cheng et al. found that rs10811661 on 9p21.3, as the neighbor SNP of rs10811656, was associated with type 2 diabetes and CAD, but it was not correlated with the severity of coronary atherosclerosis in a Chinese Han population [21]. The results of these previous studies taken together with our results suggest that further investigation is warranted to determine whether the CAD risk alleles on $9 \mathrm{p} 21.3$ are related to the severity of coronary atherosclerosis.

Our stratification analysis showed that the $\mathrm{T}$ allele was significantly associated with CAD in males. Males who carried the variant genotypes $(\mathrm{TT}+\mathrm{TC})$ had a significantly higher risk of CAD than the CC carriers. The $\mathrm{T}$ allele could be a genetic susceptibility marker for CAD in males. Valentina et al., reported that in males with breast cancer, the rs1011970/9p21.3 risk genotype was associated with HER2+ disease [22]. Lin et al. reported that chromosome $9 \mathrm{p} 21$ had a significant association with carotid atherosclerosis, especially the intima-media thickness of the internal carotid artery, in a gender-specific manner in a Chinese Han population [23]. However, little is known regarding the functional consequences of variants on $9 \mathrm{p} 21.3$, or why the variants exert a

Table 5 Association of rs10811656 with the number of diseased vessels

\begin{tabular}{|c|c|c|c|c|c|c|}
\hline SNP & Group & Genotype & Crude OR (95 \% Cl) & $P$ & Adjusted OR $(95 \% \mathrm{Cl})^{\mathrm{a}}$ & $P$ \\
\hline \multirow[t]{5}{*}{ rs10811656 } & & $\mathrm{CC} / \mathrm{CT} / \mathrm{TT}$ & & & & \\
\hline & Control & $99 / 118 / 26$ & 1 & & 1 & \\
\hline & One-vessel disease & $49 / 67 / 14$ & $1.07(0.78-1.48)$ & 0.67 & $1.14(0.82-1.60)$ & 0.42 \\
\hline & Two-vessel disease & 25/43/17 & $1.57(1.09-227)$ & 0.015 & $1.68(1.14-2.44)$ & 0.009 \\
\hline & Three-vessel disease & 29/37/17 & $1.41(0.97-2.04)$ & 0.068 & $1.60(1.10-2.33)$ & 0.02 \\
\hline
\end{tabular}


Table 6 Lipid profiles based on the rs10811656 genotypes in the CAD cases and controls

\begin{tabular}{|c|c|c|c|c|c|c|c|}
\hline \multirow[b]{2}{*}{ SNP } & \multirow[t]{2}{*}{ Characteristics } & \multicolumn{2}{|l|}{ Controls } & \multirow[t]{2}{*}{$P$} & \multicolumn{2}{|l|}{ CAD cases } & \multirow[t]{2}{*}{$P$} \\
\hline & & CC & $C T+\pi$ & & CC & $\mathrm{CT}+\pi \mathrm{T}$ & \\
\hline \multirow[t]{4}{*}{ rs10811656 } & TG (mmol/L) & $1.46 \pm 1.08$ & $2.21 \pm 6.91$ & 0.294 & $1.66 \pm 1.01$ & $1.70 \pm 2.38$ & 0.874 \\
\hline & TC (mmol/L) & $4.28 \pm 1.11$ & $4.75 \pm 2.00$ & 0.039 & $4.40 \pm 1.30$ & $4.40 \pm 1.20$ & 0.995 \\
\hline & $\mathrm{HDL}-\mathrm{C}(\mathrm{mmol} / \mathrm{L})$ & $1.16 \pm 0.27$ & $1.21 \pm 0.32$ & 0.235 & $1.15 \pm 0.26$ & $1.16 \pm 0.28$ & 0.759 \\
\hline & LDL-C (mmol/L) & $2.72 \pm 0.79$ & $2.86 \pm 0.73$ & 0.164 & $2.70 \pm 0.89$ & $2.67 \pm 0.84$ & 0.754 \\
\hline
\end{tabular}

HDL-C high density lipoprotein cholesterol, LDL-C low density lipoprotein cholesterol, TC total cholesterol, TG triglycerides

gender-specific effect on the risk of CAD. Further research will be needed to elucidate the biological significance of these associations.

We noted that among smokers, carriers of homozygous TT and heterozygous TC had an increased risk of CAD than CC genotype carriers. Our result suggests that the rs10811656 polymorphism may be factor that contributes to smoking-related CAD in a Chinese population. There was also no significant association between the rs10811656 genotypes and serum triglycerides, HDL-C and LDL-C levels in the CAD patients and controls. Therefore, our study revealed that the risk allele was associated with angiographically characterized coronary atherosclerosis, suggesting that the risk SNP exerts cell or tissue-specific effects relevant to atherosclerosis rather than affecting the traditional risk factors for CAD.

Our study has several limitations. First, selection bias and a relatively small sample size in the present study may have reduced the statistical power of the results, even though the genotype distribution of patients and controls in our study was compatible with the Hardy-Weinberg expectations. Secondly, a prospective long-term outcome study is warranted to analyze the association of polymorphism with longterm prognosis in CAD patients. Lastly, our data should be extrapolated to other regions and ethnic groups cautiously.

\section{Conclusion}

To the best of our knowledge, the present study demonstrates for the first time that the rs10811656 polymorphism is associated with increased risk of CAD in a Chinese population. Additionally, in male subjects and smokers, rs10811656 homozygous carriers of TT have an increased risk of CAD. Moreover, we found that the rs10811656 T variant is significantly correlated with SAP and angiographically characterized coronary disease severity. In order to enhance understanding of the relationship between this polymorphism and risk of CAD, further large-scale studies are required in other populations.
Acknowledgements

Not applicable.

\section{Funding}

The study was funded by grants from the National Natural Science Foundation of China (No 81270255), the Science and Technological Innovation Group of Jiangsu Higher Education Institution "Qing-Lan Project" (JX2161015030), the Priority Academic Program Development of Jiangsu Higher Education Institutions (PAPD), Shanghai Pudong New Area Science and Technology Development Fund (PKJ2014-Y09) and Shanghai Municipal Commission of Health and Family Planning Research Project (201440309).

The funders had no role in study design, data collection and analysis, decision to publish, or preparation of the manuscript.

\section{Availability of data and materials}

All data generated or analyzed during the current study are available from the corresponding author on reasonable request.

\section{Authors' contributions}

Z C conceived and designed the study, Z C and LS W interpreted the results, JJ Y analyzed the data and wrote the manuscript. JM Z, ZY X, DC $L$ participated in the laboratory tests. LS W, Z C contributed reagents/materials/analysis tools. All authors read and approved the final manuscript.

\section{Competing interests}

The authors declare that they have no competing interests.

\section{Consent for publication}

Not applicable.

\section{Ethics approval and consent to participate}

This study was approved by the Ethics Committee of Shanghai Jiao Tong University Affiliated Sixth People's Hospital, East Campus and informed consent was obtained from each participant. All authors had no access to information that could identify individual participants after data collection.

\section{Author details}

${ }^{1}$ Department of Cardiology, the First Affiliated Hospital of Nanjing Medical University, Nanjing 210029, China. ${ }^{2}$ Devision of Cardiology, The Affiliated Jiangning Hospital of Nanjing Medical University, Nanjing 211100, China. ${ }^{3}$ Department of Cardiology, Shanghai Jiao Tong University Affiliated Sixth People's Hospital, East Campus, No. 222, Huanhu Xisan Road, Pudong New Area, Shanghai 201306, China.

Received: 12 May 2016 Accepted: 27 July 2016

Published online: 09 August 2016

\section{References}

1. Roger VL, Go AS, Lloyd-Jones DM, Benjamin EJ, Berry JD, Borden WB, Bravata DM, Dai S, Ford ES, Fox CS, et al. Heart disease and stroke statistics-2012 update: a report from the American Heart Association. Circulation. 2012;125:e2-220.

2. Lian Z, Xie Y, Lu Y, Huang D, Shi H. Trends in the major causes of death in China, 1982-2010. Chin Med J (Engl). 2014;127:777-81. 
3. Zhang Y, Tian Y, Dong J, Li L, Dong Z, Deng X. Application of a multiplex SNP genotyping system in predicting genetic susceptibility to CAD in Chinese people of Han ethnicity. Med Sci Monit. 2010;16:BR384-95.

4. Motterle A, Pu X, Wood H, Xiao Q, Gor S, Ng FL, Chan K, Cross F, Shohreh B, Poston RN, et al. Functional analyses of coronary artery disease associated variation on chromosome 9p21 in vascular smooth muscle cells. Hum Mol Genet. 2012:21:4021-9.

5. Chen G, Fu X, Wang G, Liu G, Bai X. Genetic variant rs10757278 on chromosome 9p21 contributes to myocardial infarction susceptibility. Int J Mol Sci. 2015;16:11678-88.

6. Loinard C, Basatemur G, Masters L, Baker L, Harrison J, Figg N, Vilar J, Sage AP, Mallat Z. Deletion of chromosome 9p21 noncoding cardiovascular risk interval in mice alters Smad2 signaling and promotes vascular aneurysm. Circ Cardiovasc Genet. 2014;7:799-805.

7. Abrantes P, Santos MM, Sousa I, Xavier JM, Francisco V, Krug T, Sobral J, Matos M, Martins M, Jacinto A, et al. Genetic variants underlying risk of intracranial aneurysms: insights from a GWAS in Portugal. PLoS One. 2015; 10:e0133422.

8. Weiner DA, Ryan TJ, McCabe CH, Chaitman BR, Sheffield LT, Fisher LD, Tristani F. Value of exercise testing in determining the risk classification and the response to coronary artery bypass grafting in three-vessel coronary artery disease: a report from the Coronary Artery Surgery Study (CASS) registry. Am J Cardiol. 1987;60:262-6.

9. Zhang Z, Wang BJ, Guan HY, Pang H, Xuan JF. A LDR-PCR approach for multiplex polymorphisms genotyping of severely degraded DNA with fragment sizes <100 bp. J Forensic Sci. 2009;54:1304-9.

10. Almontashiri NA, Fan M, Cheng BL, Chen HH, Roberts R, Stewart AF. Interferon-gamma activates expression of p15 and p16 regardless of 9p21.3 coronary artery disease risk genotype. J Am Coll Cardiol. 2013;61:143-7.

11. Pasmant $\mathrm{E}$, Laurendeau I, Heron D, Vidaud M, Vidaud D, Bieche I. Characterization of a germ-line deletion, including the entire INK4/ARF locus, in a melanoma-neural system tumor family: identification of ANRIL, an antisense noncoding RNA whose expression coclusters with ARF. Cancer Res. 2007;67:3963-9.

12. Gianfagna F, Tamburrelli C, Vohnout B, Crescente M, Izzi B, Pampuch A, De Curtis A, Di Castelnuovo A, Cutrone A, Napoleone E, et al. Heritability, genetic correlation and linkage to the 9p21.3 region of mixed plateletleukocyte conjugates in families with and without early myocardial infarction. Nutr Metab Cardiovasc Dis. 2013;23:684-92.

13. Harismendy O, Notani D, Song X, Rahim NG, Tanasa B, Heintzman N, Ren B, Fu XD, Topol EJ, Rosenfeld MG, Frazer KA. 9p21 DNA variants associated with coronary artery disease impair interferon-gamma signalling response. Nature. 2011;470:264-8.

14. Almontashiri NA, Antoine D, Zhou X, Vilmundarson RO, Zhang SX, Hao KN, Chen $\mathrm{HH}$, Stewart AF. 9p21.3 coronary artery disease risk variants disrupt TEAD transcription factor-dependent transforming growth factor beta regulation of p16 expression in human aortic smooth muscle cells. Circulation. 2015;132:1969-78.

15. Zeng Q, Yuan Y, Wang S, Sun J, Zhang T, Qi M. Polymorphisms on chromosome 9p21 confer a risk for acute coronary syndrome in a Chinese Han population. Can J Cardiol. 2013:29:940-4.

16. Anderson JL, Horne BD, Kolek MJ, Muhlestein JB, Mower CP, Park JJ, May HT, Camp NJ, Carlquist JF. Genetic variation at the 9p21 locus predicts angiographic coronary artery disease prevalence but not extent and has clinical utility. Am Heart J. 2008;156:1155-62. e1152.

17. Chen SN, Ballantyne CM, Gotto Jr AM, Marian AJ. The 9p21 susceptibility locus for coronary artery disease and the severity of coronary atherosclerosis. BMC Cardiovasc Disord. 2009;9:3.

18. Muendlein A, Saely CH, Rhomberg S, Sonderegger G, Loacker S, Rein P, Beer $\mathrm{S}$, Vonbank A, Winder T, Drexel H. Evaluation of the association of genetic variants on the chromosomal loci 9p21.3, 6q25.1, and 2q36.3 with angiographically characterized coronary artery disease. Atherosclerosis. 2009; 205:174-80.

19. Ye S, Willeit J, Kronenberg F, Xu Q, Kiechl S. Association of genetic variation on chromosome 9p21 with susceptibility and progression of atherosclerosis: a population-based, prospective study. J Am Coll Cardiol. 2008;52:378-84.

20. Buysschaert I, Carruthers KF, Dunbar DR, Peuteman G, Rietzschel E, Belmans A, Hedley A, De Meyer T, Budaj A, Van de Werf F, et al. A variant at chromosome 9p21 is associated with recurrent myocardial infarction and cardiac death after acute coronary syndrome: the GRACE Genetics Study. Eur Heart J. 2010;31:1132-41.
21. Cheng X, Shi L, Nie S, Wang F, Li X, Xu C, Wang P, Yang B, Li Q, Pan Z, et al. The same chromosome 9p21.3 locus is associated with type 2 diabetes and coronary artery disease in a Chinese Han population. Diabetes. 2011;60:680-4.

22. Silvestri V, Rizzolo P, Scarno M, Chillemi G, Navazio AS, Valentini V, Zelli V, Zanna I, Saieva C, Masala G, et al. Novel and known genetic variants for male breast cancer risk at 8q24.21,9p21.3, 11q13.3 and 14q24.1: results from a multicenter study in Italy. Eur J Cancer. 2015;51:2289-95.

23. Lin HF, Tsai PC, Lin RT, Khor GT, Sheu SH, Juo SH. Sex differential genetic effect of chromosome 9p21 on subclinical atherosclerosis. PLoS One. 2010;5: e15124.

\section{Submit your next manuscript to BioMed Central and we will help you at every step:}

- We accept pre-submission inquiries

- Our selector tool helps you to find the most relevant journal

- We provide round the clock customer support

- Convenient online submission

- Thorough peer review

- Inclusion in PubMed and all major indexing services

- Maximum visibility for your research

Submit your manuscript at www.biomedcentral.com/submit

) Biomed Central 